\title{
Malignant Warthin's tumour: an ultrastructural study
}

\author{
LAURENCE JR BROWN, SAMUEL R APARICIO
}

From the Department of Pathology, University of Leeds, and Electronmicroscopy Unit, Clinical Sciences Building, St James's Hospital, Leeds

SUMMARY Undifferentiated malignant cells in a Warthin's tumour (adenolymphoma) were studied by light and electron microscopy. The ultrastructure of these cells indicated that they were poorly differentiated adenocarcinoma arising from the glandular component of the Warthin's tumour. Some differences between this case and previous ultrastructural studies are described.

Warthin's tumour (adenolymphoma, papillary cystadenoma lymphomatosum) is an uncommon tumour which is found predominantly in men, usually affecting the parotid gland. Malignant change is rare. ${ }^{12}$ There have been two previous ultrastructural studies, only one of which dealt with malignant change. ${ }^{34}$ The case presented here is of a malignant Warthin's tumour, which by light and electron microscopy showed some differences to previously reported material.

\section{Case report}

A 68 year old woman presented with a unilateral, symptomless, slowly growing swelling at the angle of the left side of her jaw, which had been present for two years. Further examination, including computed tomography of the head and thorax, did not show any coexistent neoplasm. Biopsy of an enlarged homolateral posterior triangle lymph node indicated a malignant Warthin's tumour, and the patient was readmitted for definitive excision. At operation the swelling had enlarged since the first examination and was a hard fixed mass covering an area measuring $3 \mathrm{~cm} \times 2 \mathrm{~cm}$ at the angle of the left jaw. Tumour cells were infiltrating into the upper posterior triangle lymph nodes, and most of the lesion was removed by a major neck dissection. The patient received postoperative radiotherapy, and two years after the initial diagnosis there has been no recurrence.

\section{METHODS}

Specimens were fixed in buffered formalin, routinely wax processed, and sections were stained with haematoxylin and eosin, periodic acid Schiff, alcian blue, Masson-Fontana, Schmorl's and for reticulin.
Representative blocks from the formalin fixed material were routinely processed for electron microscopy, and ultrathin sections were examined using a JEOL 100S transmission electron microsope at magnifications ranging from $\times 500$ to $\times 20000$.

\section{RESULTS}

Gross pathology

The specimen removed at operation was a friable and partly cystic nodule of cream coloured tissue measuring $1.8 \mathrm{~cm} \times 2.2 \mathrm{~cm} \times 1.5 \mathrm{~cm}$. No separate lymph nodes were identified.

\section{Light microscopy}

Both the biopsy specimen and the operative specimen showed the typical lymphoid and epithelial elements of an adenolymphoma but with islands of large anaplastic tumour cells within the lymphoid stroma (Fig. 1). The nuclei of the epithelial cells were mostly regular and arranged in orderly double layers, but there were a few areas where there was glandular hyperplasia leading to a cribriform pattern with anisocytosis and nuclear pleomorphism. These areas were thought to be dysplastic and therefore regarded as transitional areas between the benign and malignant components.

The cytologically malignant cells were distributed singly or arranged in irregular groups within the lymphoid stroma. These cells were large, pale, and polygonal, showing an increased nuclearcytoplasmic ratio. Nuclei were pale and vesicular, pleomorphic, had a variable chromatin pattern with large amounts of euchromatin, a high mitotic rate (11/100 high power field), and slightly eosinophilic nucleoli (Fig. 1). The cells did not stain with periodic acid Schiff, alcian blue, Masson-Fontana, or Schmorl's. Compared with the benign epithelium, the granularity and eosinophilia of the cytoplasm were less pronounced and more focally distributed within the cell. Malignant cells extended in an 


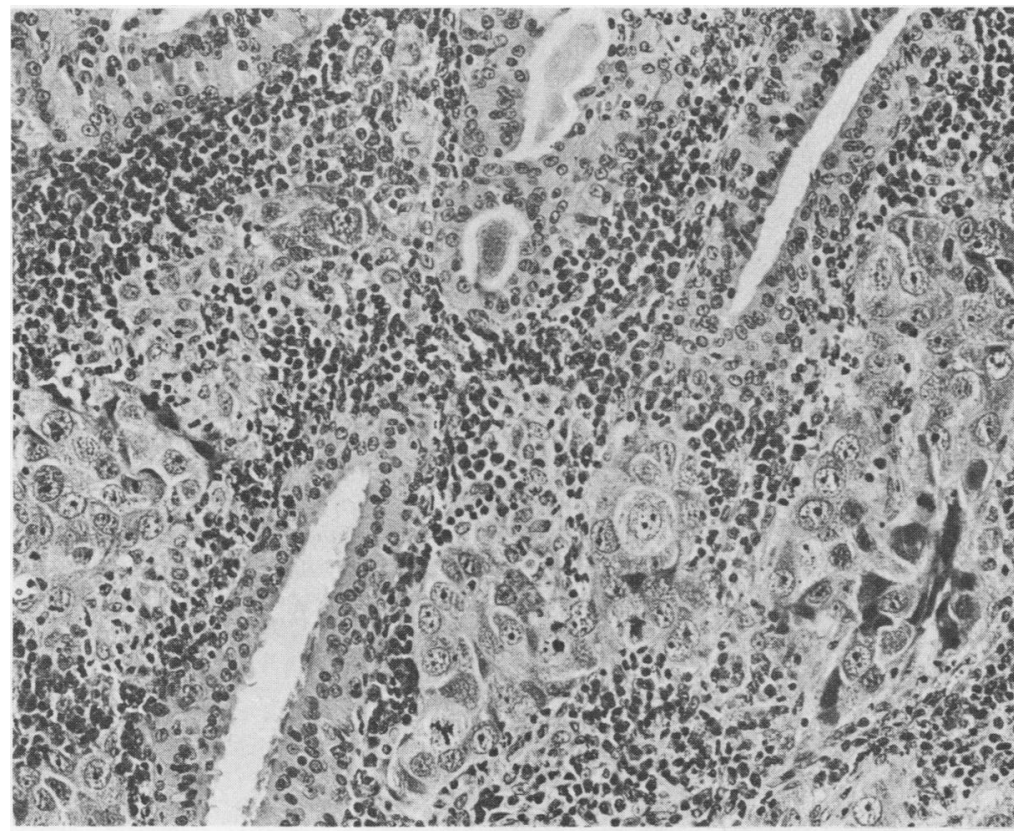

Fig. 1 Anaplastic large cells in fitrate the lymphoid stroma of the adenolymphoma; an abnormal mitosis is seen at centre bottom. Haematoxylin and eosin $\times 400$.

infiltrative pattern to the limits of the surgical excision.

\section{Electron microscopy}

The epithelial cells of the adenolymphoma were small (typically $15 \mu \mathrm{m} \times 7 \mu \mathrm{m}$ ) and regular with fairly uniform sometimes crenated nuclei $(5 \mu \mathrm{m}$ diameter) (Fig. 2). The cytoplasm was packed with numerous round or slightly oval mitochondria from $0.3 \mu \mathrm{m}$ to $1.0 \mu \mathrm{m}$ in diameter, some of which had abundant parallel linear cristae. Certain mitochondria showed aggregation of the cristae to form sheaves (Fig. 3). Other organelles seen were secretory vesicles, liposomes, lysosomes, small amounts of rough endoplasmic reticulum, and profiles of Golgi apparatus. Moderate numbers of intermediate filaments, some of which were arranged in bundles, were present. Epithelial features such as junctional complexes, a terminal web, complex interdigitating cell membranes, desmosomes, apical microvilli, and a basement membrane with hemidesmosomes were all evident (Fig. 3). Several apoptotic cells were associated with the epithelial component, but there was no evidence for two cell populations (Fig. 2).

The anaplastic cells showed some or all of the foregoing features in a variable and less organised pattern. They were much larger (up to $30 \mu \mathrm{m}$ ) and had large randomly orientated, irregularly shaped nuclei (up to $15 \mu \mathrm{m}$ diameter) (Fig. 2). Small amounts of heterochromatin were present, and there were up to two large $(2 \cdot 5 \mu \mathrm{m}$ diameter $)$ nuc- leoli. The mitochondria were variable in size and sparsely distributed in the cytoplasm but nevertheless were similar to those of the benign epithelium in that they had numerous parallel cristae which were occasionally arranged in sheaves (Fig. 4). Multiple secretory vesicles, lysosomes, and a few intracytoplasmic lumina were seen. In addition, some of the anaplastic cells contained clear lipid vacuoles, often clustered in variably sized groups (Fig. 2). Intermediate filaments and complex interdigitating cell membranes similar to those seen in the benign component were a feature of the anaplastic cells (Fig. 4). Desmosomes and poorly formed hemidesmosomes were present, but keratohyaline granules and basement membrane like material could not be identified. A few of the malignant cells were arranged into microacini (Fig. 5). These cells showed a clear apical zone, stunted microvilli, and directional organisation of the organelles.

The stroma contained more plasma cells than had been apparent on light microscopy, but no differences in the stroma associated with either the benign or malignant components could be detected (Fig. 5).

\section{Discussion}

There have been reports of the following arising in adenolymphoma: adenocarcinoma, ${ }^{5-8}$ squamous carcinoma, ${ }^{9-11}$ and mucoepidermoid carcinoma. ${ }^{12-14}$ In a recent paper Krogdal and Bretlau ${ }^{15}$ report a case of adenocarcinoma developing in an 


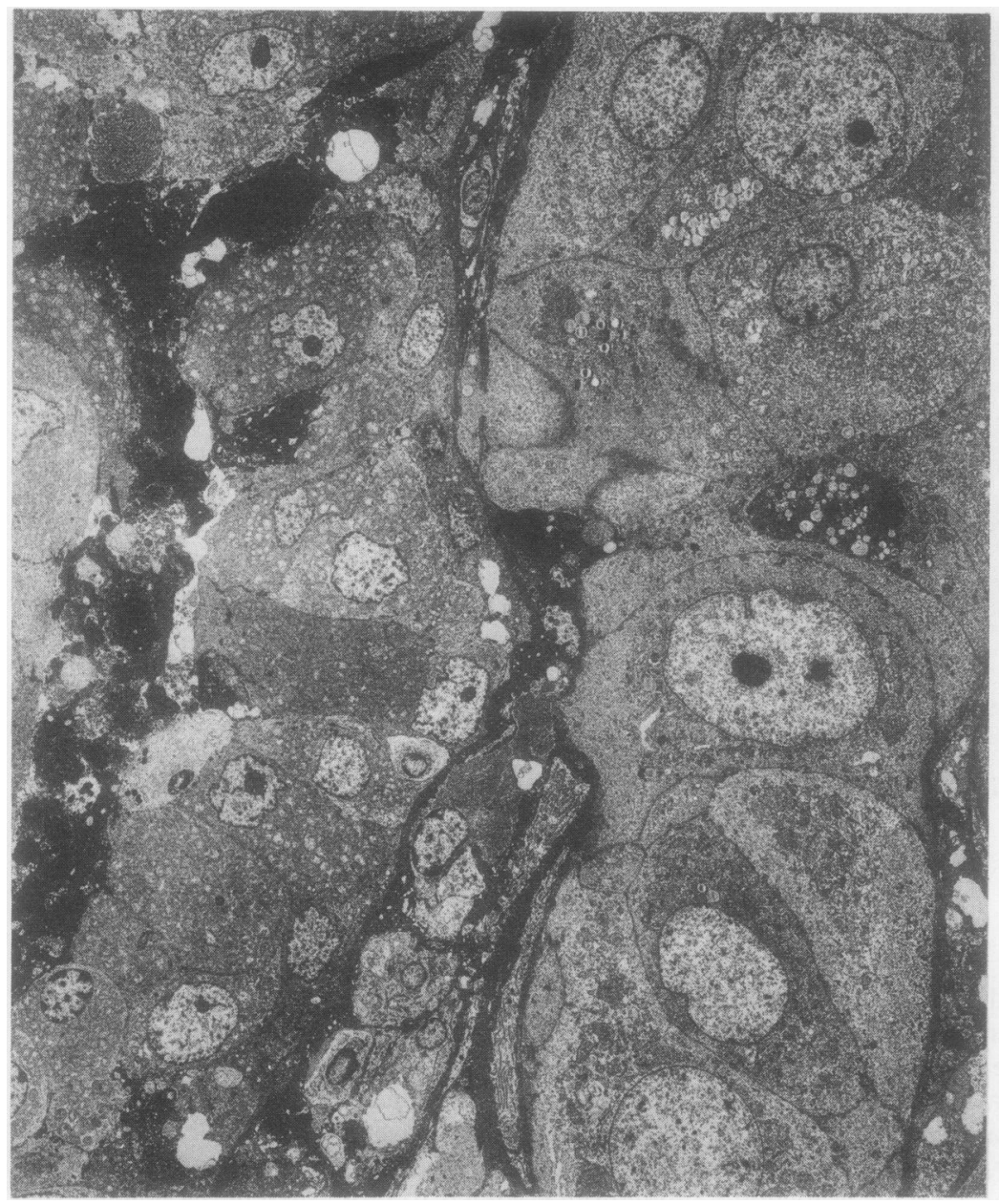

Fig. 2 Low magnification electron micrograph showing a cord of anaplastic cells adjacent to an epithelial cleft of the adenolymphoma (right of centre) containing apoptotic debris in the lumen. $\times 2000$.

adenolymphoma and give a critical view of the published work.

In the present case, light microscopy showed the typical histology of an adenolymphoma and islands of anaplastic tumour cells scattered among and infiltrating the adenolymphoma. Areas of epithelial hyperplasia and dysplasia where the cells were arranged in cribriform sheets were interpreted as a progression from benign epithelium through dysplasia to poorly differentiated carcinoma.

Of the two previous ultrastructural studies of an adenolymphoma ${ }^{34}$ only one $\mathrm{C}^{4}$ dealt with malignant change. Using electron microscopy we have shown general epithelial similarities between the benign epithelium and malignant component such as desmosomes, hemidesmosomes, interdigitating cell membranes, and microvilli. Secretory vesicles, Golgi apparatus, a terminal web, acinus formation, intracellular lumina, and directional organisation of organelles in the malignant cells are taken to indicate glandular rather than squamous differentiation. Absence of keratohyaline granules would tend to confirm this. In addition, the similarities in mitochondrial morphology, especially the arrangement of the cristae into sheaves, indicate origin from the salivary gland epithelium of the adenolymphoma.

The previous ultrastructural studies ${ }^{34}$ detailed two populations of cells forming the epithelium of the adenolymphoma. We could not confirm this, but we have described an appreciable population of apoptotic cells, which has not been reported previously.

In Ackerman's Surgical Pathology ${ }^{16}$ it is suggested that adenolymphomas are benign; this case and others, ${ }^{4-1115}$ however, show that the lesion is capable of malignant transformation, albeit rarely. The ultrastructral features strongly suggest that the 


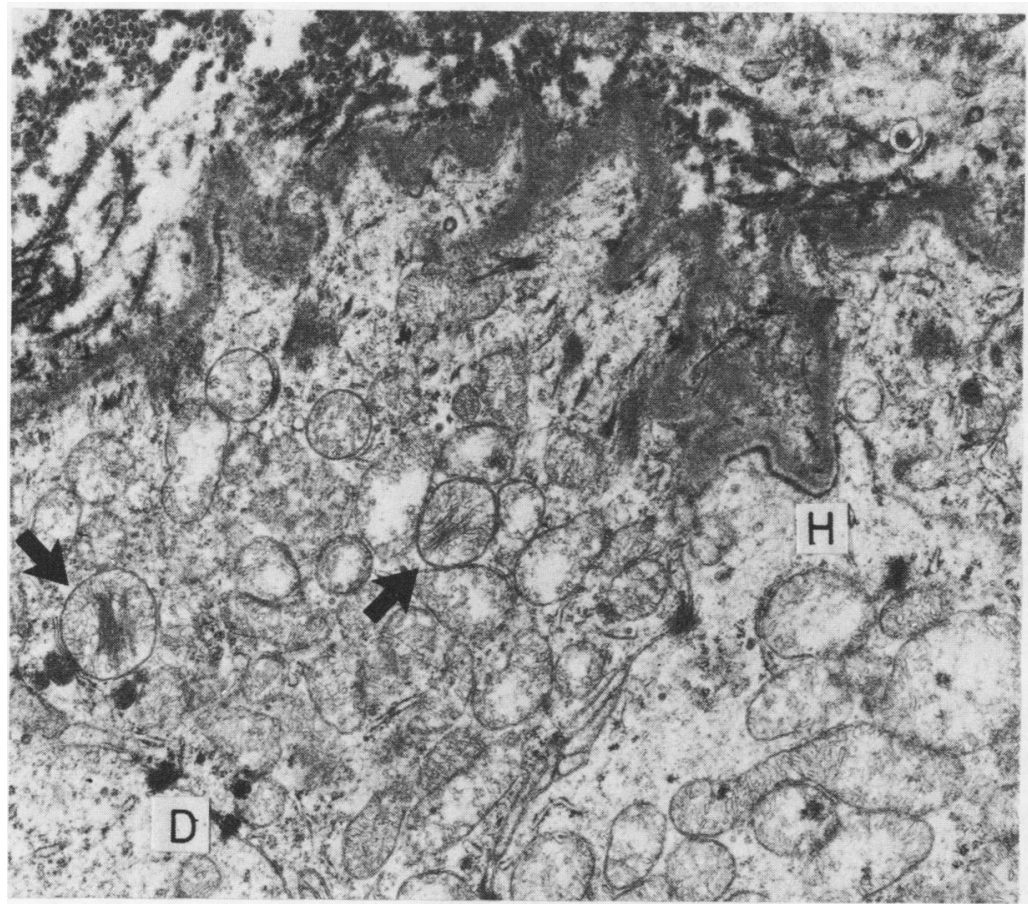

Fig. 3 Details of the cytoplasm of three benign epithelial cells, limited by a basal lamina (top). Several desmosomes $(D)$, hemidesmosomes $(H)$, and mitochondria with sheaves of cristae (arrows) are seen. $E M \times 12000$.

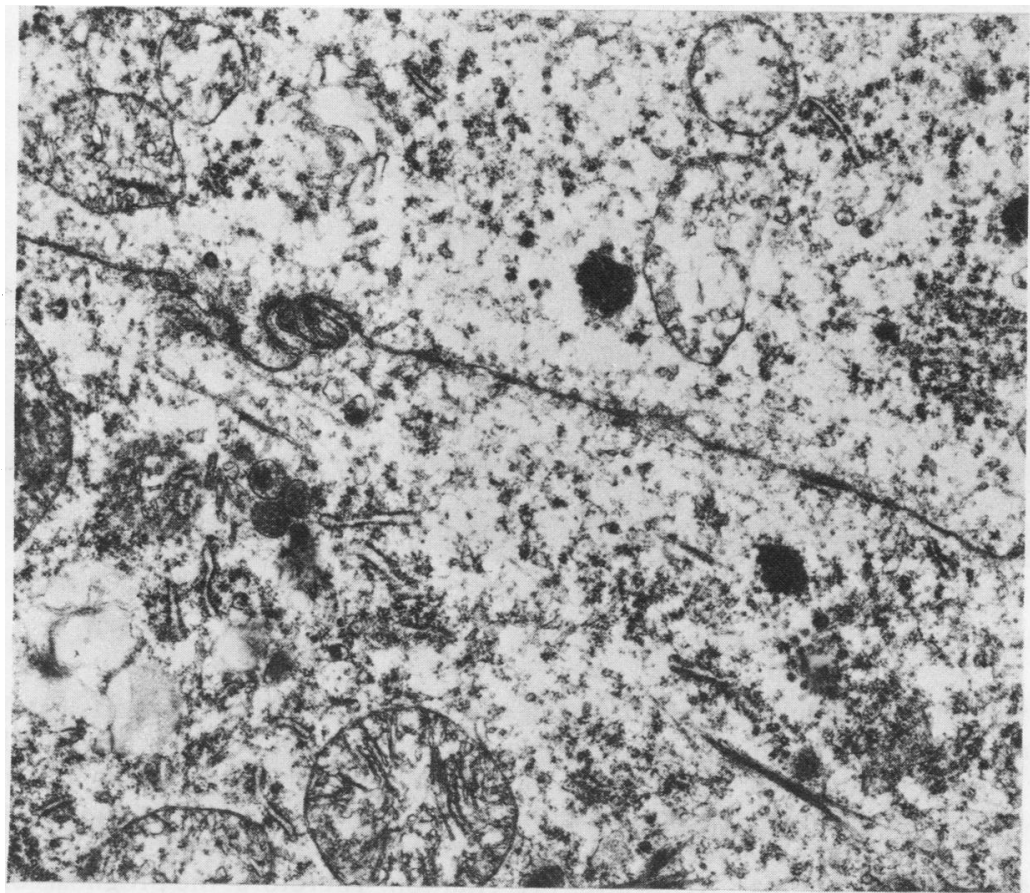

Fig. 4 Cytoplasmic details of two adjacent malignant epithelial cells. Interdigitating cell borders (left of centre) and several thin bundles of intermediate flaments are seen. The two largest mitochondria in the field show arrangement of cristae similar to that seen in Fig. 3. EM $\times 12000$. 


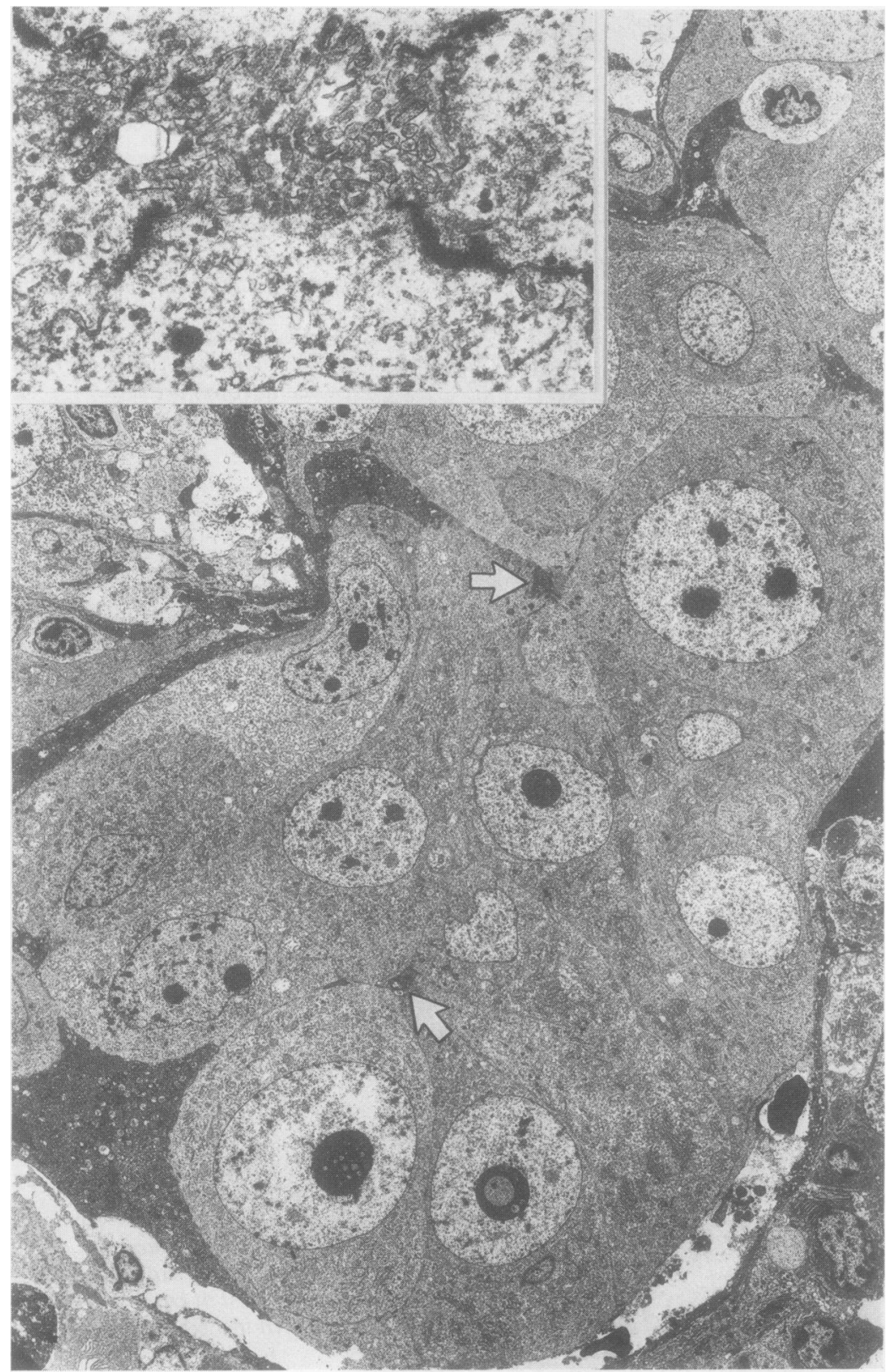

Fig. 5 Anaplastic cells showing acinar arrangement with narrow lumina at arrows. Stroma with plasma cells at bottom left. $E M \times 2000$. Inset: detail of one lumen with microvilli in the centre. $E M$ $\times 20000$.

malignant cells have derived from the glandular component and in this case have given rise to an adenocarcinoma. Moreover, unlike previous cases, ${ }^{691014}$ no other prior or coexistent primary malignancy which could have metastasised to the parotid was found. The malignant adenolymphoma itself has been said to metastasise in five reports ${ }^{5-9} 15$ and on occasion to cause death between three and 10 years $^{578}$ later. In common with several other cases described ${ }^{41-13}$ distant metastasis has not occurred in our patient and she remains well two years after presentation. Therefore, the prognosis of 
malignant adenolymphoma seems to be difficult to predict, but in this case it appears to behave in an indolent manner.

We are grateful to Professor G R Giles for allowing us to use the clinical material relevant to this case.

\section{References}

' Warthin AS. Papillary cystadenoma lymphomatosum. A rare teratoid of the parotid region. J Cancer Res 1929;13:116-25.

${ }^{2}$ Foote FW, Frazell EL. Tumours of the major salivary glands. Atlas of tumour pathology, Vol II. Washington DC: Armed Forces Institute of Pathology, 1954:129-36.

${ }^{3}$ Gavran $\mathrm{MH}$. The ultrastructure of papillary cystadenoma lymphomatosum of the parotid gland. Virchow Arch Pathol Anat 1965;338: 195-202.

${ }^{4}$ Moosavi M, Ryan CK, Schwartz S, Donnelly JA. Malignant adenolymphoma. Human Pathol 1980;11:80-3.

s Reubner B, Bramall JL. Malignant papillary cystadenoma lymphomatosum. AMA Arch Pathol 1980;69:110-7.

- Little JW, Rickles NH. Malignant papillary cystadenoma lymphomatosum. Cancer 1961;18:790-4.

${ }^{7}$ Döbrössy L, Ronay D, Molnár C. Malignant papillary cystadenoma lymphomatosum. Oncology 1972;26:457-65.
${ }^{8}$ Kessler E, Koznizky IL, Schinder J. Malignant Warthin's tumour. Oral Surg 1977;43:111-5.

- De La Pava S, Knutson GH, Moktar F, Pickren JW. Squamous cell carcinoma arising in a Warthin's tumour of the parotid gland. Cancer 1965;18:790-4.

${ }^{10}$ Assor D. Bilateral carcinoma of the parotid, one cancer arising within a Warthin's tumour. Am J Clin Pathol 1974;61:270-4.

" Baker M, Yuzon D, Baker B. Squamous cell carcinoma arising in benign adenolymphoma (Warthin's tumour) of the parotid gland. J Surg Oncol 1980;15:7-10.

12 Tanaka N, Chen WC. A case of bilateral papillary cystadenoma lymphomatosum (Warthin's tumour) of the parotid complicated with muco-epidermoid tumour. Gan 1953;44:224-31.

${ }^{13}$ Gadient SE, Kalfayan B. Muco-epidermoid carcinoma arising within a Warthin's tumour. Oral Surg 1975;40:391-8.

${ }^{14}$ Lumeran H, Freedman P, Caracciolo P, Remigio PS. Synchronous malignant muco-epidermoid tumour of the parotid gland and Warthin's tumour in adjacent lymph node. Oral Surg 1975;43:953-8.

is Krogdahl AS, Bretlau P. Malignant transformation of adenolymphomas. Ann Otol Rhinol 1983;92:49-52.

${ }^{16}$ Rosai J. Ackerman's surgical pathology, 6th ed. London: CV Mosby Company, 1981:577-9.

Requests for reprints to: Dr LJR Brown, Department of Pathology, University of Leeds, Leeds, England 